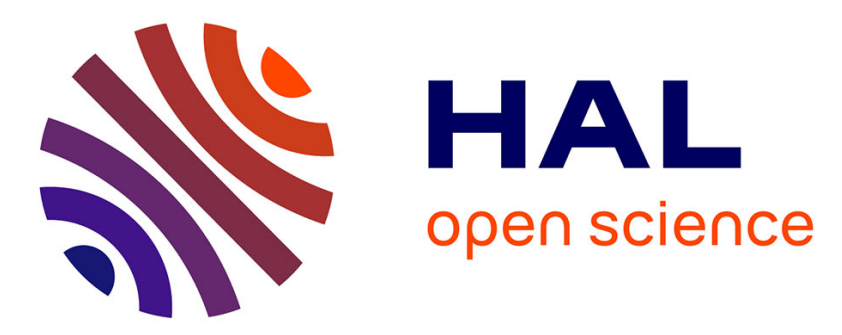

\title{
A simple and efficient numerical model for thermal contact resistance based on diffuse interface immersed boundary method
}

\author{
A Aalilija, Ch.-A. Gandin, E Hachem
}

\section{- To cite this version:}

A Aalilija, Ch.-A. Gandin, E Hachem. A simple and efficient numerical model for thermal contact resistance based on diffuse interface immersed boundary method. International Journal of Thermal Sciences, 2021, 166, 10.1016/j.ijthermalsci.2020.106817 . hal-03448801

\author{
HAL Id: hal-03448801 \\ https://hal.science/hal-03448801
}

Submitted on 27 Nov 2021

HAL is a multi-disciplinary open access archive for the deposit and dissemination of scientific research documents, whether they are published or not. The documents may come from teaching and research institutions in France or abroad, or from public or private research centers.
L'archive ouverte pluridisciplinaire HAL, est destinée au dépôt et à la diffusion de documents scientifiques de niveau recherche, publiés ou non, émanant des établissements d'enseignement et de recherche français ou étrangers, des laboratoires publics ou privés. 


\title{
A simple and efficient numerical model for thermal contact resistance based on diffuse interface immersed boundary method
}

\author{
A. Aalilija, Ch.-A. Gandin, E. Hachem \\ MINES ParisTech, PSL - Research University, Centre for material forming (CEMEF), CNRS UMR 7635, CS 10207, 1 rue \\ Claude Daunesse, 06904 Sophia Antipolis Cedex, France
}

\begin{abstract}
Thermal contact resistance between two adjacent materials is one of the major concerns in a large variety of practical situations in heat transfer. The presence of this resistance yields a temperature jump across the interface. Due to this discontinuity, numerical modelling of thermal contact resistance runs into difficulties. In this work, we propose a simple and efficient numerical model to account for thermal contact resistance at immersed interfaces in multi-material heat transfer. The present model is based on the diffuse interface method. The effect of contact resistance between two adjacent materials is accounted for in the mixing laws of the thermal conductivity and the heat capacity. The model is validated through different test cases, including both steady and unsteady conduction, and applied to simulation of chill cooling and solidification of a steel sample on board the International Space Station.
\end{abstract}

Keywords: Conjugate heat transfer, thermal contact resistance, diffuse interface immersed boundary, level-set, mixing law, finite element method

\section{Introduction}

An interface between two adjacent bodies is perfect if the real contact occurs in every point of the contacting surfaces. In this ideal case, the temperature and the heat flux are continuous across the interface. In most practical situations, the contacting surfaces are rough and have microscopic asperities. As a result, the mechanical contact occurs only at several microscopic spots interspersed with gaps or gas cavities of poor heat transfer capability. Therefore, the heat flux from the hot body to the cold one is constrained to pass through the real contact spots, which represent only a small fraction of the total interface area. In this case, we say that the interface exhibits a Thermal Contact Resistance. At the macroscopic scale, the existence of a thermal contact resistance appears as a temperature jump across the interface. The thermal contact resistance can occur at solid-solid interfaces [1] but also at liquid-solid interfaces [2]. Many physical systems involve a mechanical combination of two or several materials. Therefore, thermal contact resistance can occur in a wide range of applications such as additive manufacturing [3], electronic packaging [4], nuclear reactors [5], hypersonic flights [6], internal combustion engines [7], die casting [8] and injection moulding [9].

The use of numerical simulation to predict different aspects of heat transfer in the industry is becoming an important tool to help to improve the performance and the efficiency of industrial processes. Numerical modelling of heat transfer in a multi-material domain, including interfaces, has long been addressed in the

Email addresses: ayoub.aalilija@mines-paristech.fr (A. Aalilija), charles-andre.gandin@mines-paristech.fr (Ch.-A. Gandin), elie.hachem@mines-paristech.fr (E. Hachem) 
literature $[10,11,12,13,14]$. However, accounting for the thermal contact resistance is still a numericallychallenging task due to the temperature discontinuity that it involves. Consequently, additional numerical efforts are needed to handle the discontinuities at the interfaces. For instance, in the classical finite element method, the meshing of the interface is required and has to conform to the adjacent volume parts. Moreover, appropriate surface elements must be constructed. For complex geometries, this is challenging [15, 16]. To circumvent this challenge, some authors built their thermal contact resistance modelling on the extended finite element method (XFEM) [16, 17, 18]. Moreover, numerical models of thermal contact resistance available in the literature focus mostly on the steady state conduction $[19,20,21,22,17]$. Only a few studies have addressed the thermal contact resistance in transient conduction [15].

In this work, we derive a simple and efficient numerical model to account for thermal contact resistance in time-dependent multi-material heat transfer problems for classical finite element method. Our numerical framework is based on the diffuse-interface immersed-boundary approach. The interface is implicitly represented by the Level-Set function. The principle of this model is to represent the contact imperfections by an intermediate thin material between the contacting bodies. Consequently, appropriate mixing laws will consistently be derived to mix the properties of the two materials together with the intermediate material. Accordingly, new formulae for mixing heat capacities and thermal conductivities will be derived. The novelty of this work lies in the fact that the proposed model does not require any additional implementation compared to the solver of perfect contact thermal transfer. The change is only made in the mixing laws of thermal properties. Furthermore, no special treatment of temperature discontinuities is needed because the temperature jump is smoothed along the mixing zone.

The present work starts from the weak formulation of heat conduction in a two-material domain. The level set method is introduced. The mixing laws for perfect contact are recalled. The modelling of thermal contact resistance is presented. Then, the mixing laws for thermal conductivity and specific heat are derived. The effectiveness and accuracy of the proposed model are assessed through different benchmarks for both steady-state and transient conduction. The numerical results are compared to analytical solutions, a numerical reference case and an experimental test of chill cooling and solidification of steel droplet in microgravity on board the International Space Station

\section{Governing equations and modelling}

This section is devoted to the mathematical formulation of heat conduction involving two materials in contact and the resolution methodology based on the monolithic formulation of the energy conservation equation using the diffuse interface immersed boundary method. The thermal contact resistance modelling is illustrated via a one-dimensional case of two adjacent slabs. A general 3D unsteady heat conduction is formulated by the level-set function. Finally, a generalised formulation for a multi-domain system will be presented.

\subsection{Two-domain formulation}

Consider a computational domain $\Omega$ containing two materials indexed by 1 and $2\left(\Omega=\Omega_{1} \cup \Omega_{2}\right)$ separated by an interface $\Gamma_{12}$. The heat transfer operated by conduction is described by the following set of equations:

$$
\begin{array}{ll}
C_{p_{1}} \frac{\partial T_{1}}{\partial t}=\nabla \cdot\left(k_{1} \nabla T_{1}\right), & \boldsymbol{x} \in \Omega_{1} \\
C_{p_{2}} \frac{\partial T_{2}}{\partial t}=\nabla \cdot\left(k_{2} \nabla T_{2}\right), & \boldsymbol{x} \in \Omega_{2}
\end{array}
$$

where $C_{p_{i}}=\rho_{i} c_{p_{i}}$ is the volumetric specific heat, product of the density, $\rho_{i}$, and the specific heat per unit mass, $c_{p_{i}} ; k_{i}$ is the thermal conductivity and $T_{i}(\boldsymbol{x}, t)$ is the temperature field, respectively in $(\mathrm{i}=1) \Omega_{1}$ and 
$(\mathrm{i}=2) \Omega_{2}$. At the interface $\Gamma_{12}$, several boundary conditions can be described. First, we assume the absence of any heat source at $\Gamma_{12}$, a situation that can occur when, for instance, an electric current flows through the contact surfaces, thus generating heat by energy dissipation. Under the latter assumption, there is continuity of the normal heat flux across $\Gamma_{12}$, such as :

$$
q_{12}=-k_{1} \nabla T_{1} \cdot \boldsymbol{n}_{12}=-k_{2} \boldsymbol{\nabla} T_{2} \cdot \boldsymbol{n}_{12}, \quad \boldsymbol{x} \in \Gamma_{12}
$$

where $\boldsymbol{n}_{12}$ is the unit normal vector to $\Gamma_{12}$ pointing at $\Omega_{2}$. If the interface is thermally resistive, i.e. a thermal contact resistance $R_{t h} \neq 0$ is posing a barrier to heat transfer at the interface $\Gamma_{12}$, a macro-scale temperature jump occurs at the interface, such as:

$$
\llbracket T \rrbracket=-R_{t h} q_{12} \quad \boldsymbol{x} \in \Gamma_{12}
$$

where $\llbracket \star \rrbracket=(\star)_{2}-(\star)_{1}$ means the jump across the interface $\Gamma_{12}$.

We consider the Dirichlet and Neumann boundary conditions prescribed on the two complementary and disjoints portions of $\partial \Omega$, denoted by $\partial \Omega_{D i r}$ and $\partial \Omega_{N e u}$ as follows:

$$
\left\{\begin{aligned}
\boldsymbol{q .} \cdot \boldsymbol{n} & =q_{B}, \quad \boldsymbol{x} \in \partial \Omega_{N e u} \\
T & =T_{B}, \quad \boldsymbol{x} \in \partial \Omega_{D i r}
\end{aligned}\right.
$$

where $\boldsymbol{n}$ is the outward normal vector to $\partial \Omega_{N e u}$.

Two different resolution strategies could be adopted to deal with this multi-material heat transfer problem. The first, called partitioned resolution, consists of considering two different meshes for each domain and solve two equations with the associated boundary conditions separately. The global solution is then constructed by a suitable assembly method. However, during the assembly, the coordination between the meshes can become complicated or even sometimes infeasible. The second strategy, which we use in this work, is called monolithic resolution. It consists of solving one equation over the whole computational domain in a single global mesh. The principle of the monolithic formulation is to treat the two sub-domains as one with variable properties $k(\boldsymbol{x})$ and $C_{p}(\boldsymbol{x})$.

To formulate the problem in a monolithic way, we write the weak formulation of each subdomain. Then, we construct a unified formulation for the global domain by summing the contribution of each subdomain. The weak formulation associated with equations (1) and boundary conditions (4) is given as follows:

$$
\begin{gathered}
\int_{\Omega_{1}} C_{p_{1}} \frac{\partial T_{1}}{\partial t} w_{1} d V+\int_{\Omega_{1}} k_{1} \nabla T_{1} \cdot \nabla w_{1} d V=\int_{\Gamma_{12}} k_{1} \nabla T_{1} \cdot \boldsymbol{n}_{12} w_{1} d S+\int_{\partial \Omega_{N e u} \cap \partial \Omega_{1}} q_{B} w_{1} d S \\
\int_{\Omega_{2}} C_{p_{2}} \frac{\partial T_{2}}{\partial t} w_{2} d V+\int_{\Omega_{2}} k_{2} \boldsymbol{\nabla} T_{2} \cdot \nabla w_{2} d V=\int_{\Gamma_{12}}-k_{2} \nabla T_{2} \cdot \boldsymbol{n}_{12} w_{2} d S+\int_{\partial \Omega_{N e u} \cap \partial \Omega_{2}} q_{B} w_{2} d S
\end{gathered}
$$

where $w_{1}$ and $w_{2}$ are test functions for the subdomains $\Omega_{1}$ and $\Omega_{2}$, respectively. Using the flux continuity condition (2) and considering the presence of a thermally resistive interface giving rise to a temperature jump at $\Gamma_{12}$ (equation (3)), the general formulation over domain $\Omega$ is obtained:

$$
\int_{\Omega} C_{p}(\boldsymbol{x}) \frac{\partial T}{\partial t} w d V+\int_{\Omega} k(\boldsymbol{x}) \boldsymbol{\nabla} T \cdot \nabla w d V+\int_{\Gamma_{12}} \frac{\llbracket T \rrbracket}{R_{t h}} \llbracket w \rrbracket d S=\int_{\partial \Omega_{N e u}} q_{B} w d S
$$

It is worth noting that for perfect contact at $\Gamma_{12}$, the temperature jump $\llbracket T \rrbracket$ vanishes so the monolithic formulation simply becomes:

$$
\int_{\Omega} C_{p}(\boldsymbol{x}) \frac{\partial T}{\partial t} w d V+\int_{\Omega} k(\boldsymbol{x}) \nabla T \cdot \nabla w d V=\int_{\partial \Omega_{N e u}} q_{B} w d S
$$


As we can observe from the latter two equations, the definition of variable properties as well as the third term in the left-hand side of equation (6) requires a numerical method to localise the boundary between the two sub-domains. There is a variety of methods to fulfil this task. Here, we opt for the level set method which has many attractive properties in studying interfacial problems with complex geometries. The level set method is based on an implicit representation of the interface via a signed distance function:

$$
\phi(\boldsymbol{x}, t)=\left\{\begin{array}{rll}
d\left(\boldsymbol{x}, \Gamma_{12}\right) & \text { if } & \boldsymbol{x} \in \Omega_{1} \\
0 & \text { if } & \boldsymbol{x} \in \Gamma_{12} \\
-d\left(\boldsymbol{x}, \Gamma_{12}\right) & \text { if } & \boldsymbol{x} \in \Omega_{2}
\end{array}\right.
$$

\section{where $d\left(\boldsymbol{x}, \Gamma_{12}\right)$ denotes the geometrical distance between the position $\boldsymbol{x}$ and the interface $\Gamma_{12}$.}

Furthermore, the discontinuity of interface properties, as well as the temperature discontinuity resulting from thermal contact resistance, are numerically challenging. It is, therefore, necessary to have a robust numerical method capable of handling high property ratios and capturing the temperature jump across the interface accurately. For this reason, Yvonnet et. al. [17] proposed a numerical procedure for thermal contact resistance at sharp interfaces, based on XFEM and the level set method.

In the present work, we use a different numerical approach with the classical Finite Element Method. Our model is based on the diffuse-interface approach, which consists in introducing an artificial region around the interface of fixed thickness $2 \varepsilon$ comparable to the mesh size in the vicinity of the interface. In this region, the transition of properties from one domain to the other occurs continuously and smoothly. The way the transition occurs is described by the mixing law. The objective of this study is not solving the equation (6) for thermal contact resistance. Instead, we look for a simple and efficient solution so as to keep equation (7) for both perfect and imperfect interfaces while finding mixing laws which account for the effect of a thermal contact resistance.

\subsection{Mixing laws for perfect contact}

For a perfect contact, it is well-known in the literature [23] that a harmonic mixing law for thermal conductivity ensures the conservation of heat flux across the transition zone.

$$
k(\phi)=\left(\frac{H(\phi)}{k_{1}}+\frac{1-H(\phi)}{k_{2}}\right)^{-1}
$$

where $H$ is a smooth Heaviside function which indicates the presence of domain $\Omega_{1}$. We choose here a sinusoidal smoothing function of the signed distance function $\phi$ (equation (8)):

$$
H(\phi)= \begin{cases}1 & \text { if } \quad \phi>\varepsilon \\ 0 & \text { if } \phi<-\varepsilon \\ \frac{1}{2}\left[1+\frac{\phi}{\varepsilon}+\frac{1}{\pi} \sin \left(\frac{\pi \phi}{\varepsilon}\right)\right] & \text { if } \quad|\phi| \leq \varepsilon\end{cases}
$$

Note that the harmonic mixing law of the thermal conductivity will be retrieved in the section dedicated to the mixing law for thermal contact resistance in the next section.

As for volumetric specific heat, the arithmetic mixing law is used:

$$
C_{p}(\phi)=C_{p_{1}} H(\phi)+C_{p_{2}}(1-H(\phi))
$$




\subsection{Mixing laws for thermal contact resistance}

The basic idea of the present modelling of thermal contact resistance is to consider that in a micro-scale the imperfections of contact can be modelled by a thin layer of thickness $e$ separating $\Omega_{1}$ and $\Omega_{2}$. This intermediate domain, denoted by $\Omega_{G}$ (G for Gap), is considered as a third material of properties $k_{G}$ and $C_{p_{G}}$ as shown in figure 1 . Following this representation, we intend to find physically-appropriate mixing laws for both $k$ and $C_{p}$ inside the mixing zone including the effect of the gap layer. For the sake of clarity, we derive the mixing law in $1 \mathrm{D}$, and then we extend the formulation to higher dimensions using the level-set function.

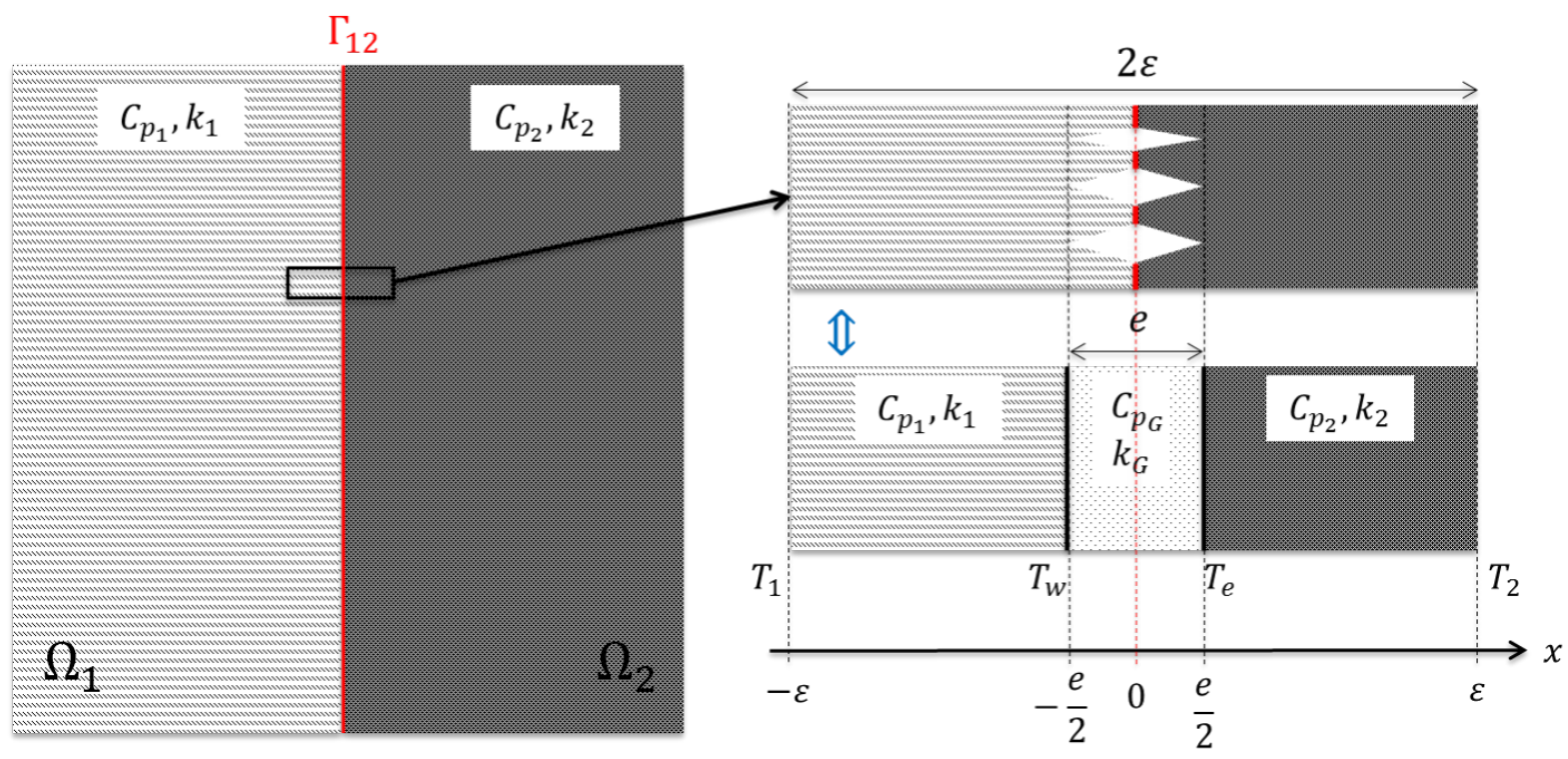

Figure 1: Schematic of two materials in contact, $\Omega_{1}$ and $\Omega_{2}$, forming a thermally resistant interface $\Gamma_{12}$ due to the presence of a gap layer, $\Omega_{G}$, with thickness $e$.

\subsubsection{Thermal conductivity mixing law}

For thermal conductivity, we limit our analysis to steady state conduction in which the specific heat of the materials does not play a role. Consider the steady state temperature field $T(x, t \rightarrow \infty)=T_{s}(x)$ in the contact region centred within $x \in[-\varepsilon, \varepsilon]$ shown in figure 1. $T_{s}$ is piecewise linear such as:

$$
T_{s}(x)=\left\{\begin{array}{rll}
T_{1}+a_{1}(x+\varepsilon) & \text { if } & x \in[-\varepsilon,-e / 2] \\
T_{w}+a_{G}(x+e / 2) & \text { if } & x \in[-e / 2, e / 2] \\
T_{2}+a_{2}(x-\varepsilon) & \text { if } & x \in[e / 2, \varepsilon]
\end{array}\right.
$$

where $a_{1}=\frac{1}{k_{1}}\left(\frac{T_{2}-T_{1}}{\frac{\varepsilon-e / 2}{k_{1}}+\frac{e}{k_{G}}+\frac{\varepsilon-e / 2}{k_{2}}}\right), a_{G}=\frac{k_{1}}{k_{G}} a_{1}$ and $a_{2}=\frac{k_{1}}{k_{2}} a_{1}$. We suppose that the mixing zone of thickness $2 \varepsilon$ has equivalent properties $k_{e q}$ and $C_{p_{e q}}$. Therefore, the heat flux across the mixing zone is given by:

$$
q_{s}=k_{e q} \frac{T_{2}-T_{1}}{2 \varepsilon} .
$$

On the other hand, the heat flux in domain $\Omega_{1}$ is:

$$
q_{s}=k_{1} a_{1}=\frac{T_{2}-T_{1}}{\frac{\varepsilon-e / 2}{k_{1}}+\frac{e}{k_{G}}+\frac{\varepsilon-e / 2}{k_{2}}} .
$$


Consequently, the heat flux conservation yields:

$$
\frac{2 \varepsilon}{k_{e q}}=\frac{\varepsilon-e / 2}{k_{1}}+\frac{e}{k_{G}}+\frac{\varepsilon-e / 2}{k_{2}} .
$$

We assume that the thickness of the gap is much smaller than the one of the mixing zone, $e \ll \varepsilon$, and that the thermal conductivity of the gap is negligible comparing to $k_{1}$ and $k_{2}, k_{G} \ll k_{i \in\{1,2\}}$. Hence, the equivalent thermal conductivity reads:

$$
\frac{1}{k_{e q}} \approx \frac{1}{2 k_{1}}+\frac{1}{2 k_{2}}+\frac{R_{t h}}{2 \varepsilon}
$$

where $R_{t h}=\frac{e}{k_{G}}$ is the thermal contact resistance with unit $K \cdot W^{-1} \cdot m^{2}$.

Using the level set function for generalisation, the idea now is to find a formula for the thermal conductivity $k(\phi)$ which links in a continuous way the region $\phi \leq-\varepsilon$ where $k=k_{1}$ and the region $\phi \geq \varepsilon$ where $k=k_{2}$ respecting that the harmonic mean of $k$ in the transition zone is:

$$
\frac{1}{2 \varepsilon} \int_{-\varepsilon}^{\varepsilon} \frac{1}{k(\phi)} d \phi=\frac{1}{k_{e q}}
$$

These conditions lead to the following mixing low for the thermal conductivity:

$$
k(\phi)=\left(\frac{H(\phi)}{k_{1}}+\frac{1-H(\phi)}{k_{2}}+R_{t h} \delta(\phi)\right)^{-1}
$$

where $\delta$ is the smooth Dirac function derived from equation (10):

$$
\delta(\phi)=\left\{\begin{array}{lll}
\frac{1}{2 \varepsilon}\left[1+\cos \left(\frac{\pi \phi}{\varepsilon}\right)\right] & \text { if } & |\phi| \leq \varepsilon \\
0 & \text { if } & |\phi|>\varepsilon
\end{array}\right.
$$

For $R_{t h}=0$, equation (18) gives the classical harmonic mixing law for perfect contact.

\subsubsection{Volumetric specific heat mixing law}

Unlike thermal conductivity, volumetric specific heat is an extensive property. Therefore, the equivalent volumetric specific heat in the mixing zone can be obtained by the volume-average of the heat capacities over the mixing zone. This leads to the following equation:

$$
\begin{aligned}
C_{p_{e q}} & =\frac{\varepsilon-e / 2}{2 \varepsilon} C_{p_{1}}+\frac{\varepsilon-e / 2}{2 \varepsilon} C_{p_{2}}+\frac{e}{2 \varepsilon} C_{p_{G}} \\
& =\bar{C}_{p}\left(1-\frac{e}{2 \varepsilon}\right)+\frac{e}{2 \varepsilon} C_{p_{G}}
\end{aligned}
$$

where $\bar{C}_{p}=\left(C_{p_{1}}+C_{p_{2}}\right) / 2$. With the assumption that $e \ll 2 \varepsilon$

$$
C_{p_{e q}} \approx \bar{C}_{p}+\frac{e}{2 \varepsilon} C_{p_{G}}
$$

Using a condition equivalent to equation (17) for the volumetric specific heat, the mixing law using the level set reads:

$$
C_{p}(\phi)=C_{p_{1}} H(\phi)+C_{p_{2}}(1-H(\phi))+e C_{p_{G}} \delta(\phi)
$$


The mixing law (22) for volumetric specific heat requires the knowledge of the values of the thickness and the volumetric specific heat of the gap. In some problems, these values may be unknown. For this reason, we will show, through numerical simulations of unsteady conduction, cases in which the additional term with gap properties is not required. Three different cases exist, $C_{p_{G}} \ll \bar{C}_{p}, C_{p_{G}}=\mathcal{O}\left(\bar{C}_{p}\right)$ and $C_{p_{G}} \gg \bar{C}_{p}$. In each case, equations (11) and (22) will be tested and compared to a reference case.

\subsection{Multi-domain formulation}

Let $\Omega$ be a computational domain composed of $N$ subdomains $\Omega_{i}, i \in 1, \ldots, N$ with $C_{p_{i}}$ the volumetric specific heat and $k_{i}$ the thermal conductivity. We still consider that thermal transfer is governed only by heat conduction. The energy conservation equation in each domain $\Omega_{i}$ writes:

$$
C_{p_{i}} \frac{\partial T_{i}}{\partial t}=\nabla \cdot\left(k_{i} \nabla T_{i}\right), \quad \boldsymbol{x} \in \Omega_{i}
$$

Following the work in section 2.1, the multi-domain weak formulation of this problem writes:

$$
\int_{\Omega} C_{p} \frac{\partial T}{\partial t} w d V+\int_{\Omega} k \nabla T \cdot \nabla w d V=\int_{\partial \Omega_{N e u}} q_{B} w d S
$$

with the following expressions for the equivalent properties $C_{p}$ and $k$ :

$$
\begin{aligned}
k & =\left(\sum_{1 \leq i \leq N} \frac{H_{i}}{k_{i}}+\sum_{1 \leq i<j \leq N} R_{t h i j} \delta_{i j}\right)^{-1} \\
C_{p} & =\sum_{1 \leq i \leq N} C_{p_{i}} H_{i}+\sum_{1 \leq i<j \leq N} e_{i j} C_{p_{G_{i j}}} \delta_{i j}
\end{aligned}
$$

where $H_{i}$ is the smooth Heaviside function associated to the subdomain $\Omega_{i}$ and $\delta_{i j}$ is the Dirac function located at the interface $\Gamma_{i j}$ separating $\Omega_{i}$ and $\Omega_{j}(j \neq i)$. At interface $\Gamma_{i j}, R_{t h i j}$ is the value of the thermal contact resistance, $e_{i j}$ is the thickness of the gap and $C_{p_{G_{i j}}}$ is its volumetric specific heat.

\section{Results and discussion}

\subsection{Steady conduction}

\subsubsection{Planar interface benchmark}

In this example, we propose a benchmark with a planar and resistive interface. The problem geometry and mesh are illustrated in figure 2 .

By considering the steady state in each domain, boundary conditions $T\left(X=X_{1}\right)=T_{B 1}$ and $T(X=$ $\left.X_{2}\right)=T_{B 2}$, the continuity of normal flux $q_{s}$ across the interface located at $X=\xi$ and the one-dimensional jump condition $\llbracket T(X=\xi) \rrbracket=-R_{t h} q_{s}(X=\xi)$, we obtain the following exact solution:

$$
T(X)=\left\{\begin{array}{lll}
\frac{q_{s}}{k_{1}}\left(X-X_{1}\right)+T_{B 1} & \text { if } & X \in\left[X_{1}, \xi\right] \\
\frac{q_{s}}{k_{2}}\left(X-X_{2}\right)+T_{B 2} & \text { if } & x \in\left[\xi, X_{2}\right]
\end{array}\right.
$$

where $q_{s}=\left(T_{B 2}-T_{B 1}\right) /\left(\frac{\xi-X_{1}}{k_{1}}+R_{t h}+\frac{X_{2}-\xi}{k_{2}}\right)$.

The boundaries are positioned at $X_{1}=-1 \mathrm{~m}$ and $X_{2}=1 \mathrm{~m}$. The temperatures at these two boundaries are $T_{B 1}=0 \mathrm{~K}$ and $T_{B 2}=1 \mathrm{~K}$. The other boundaries of the computational domain are adiabatic $\left(q_{B}=0\right)$. 


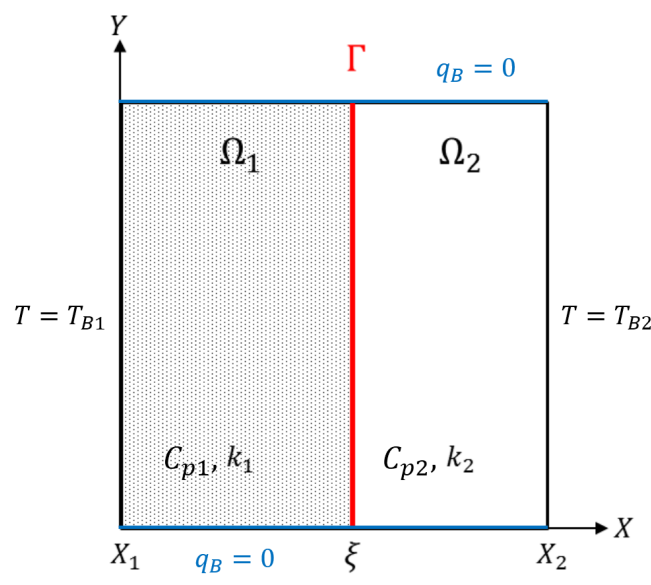

(a) Geometry

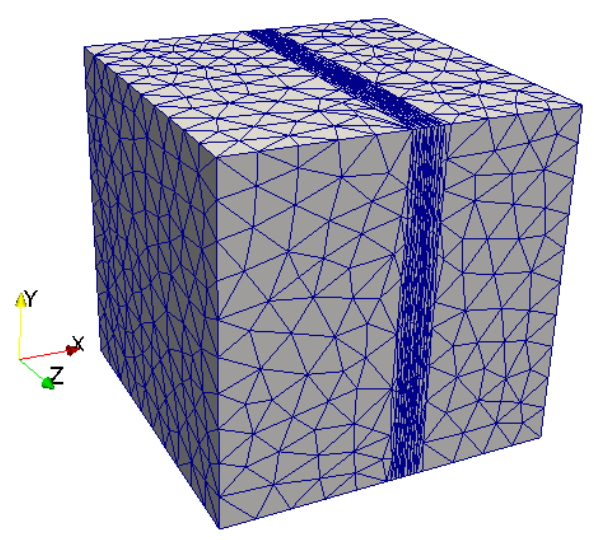

(b) Mesh

Figure 2: Setup for numerical simulations using a 1D heat flow configuration between the two materials in contacts, as defined in figure 1.

The interface position is $\xi=0.09 \mathrm{~m}$. The mesh is anisotropic in the mixture zone. The characteristic mesh sizes in this region are $h_{\perp}=0.02 \mathrm{~m}$ in the direction perpendicular to the interface (also the direction of the heat flux) and $h_{\|}=0.2 \mathrm{~m}$ in the direction parallel to the interface. Outside the mixture zone, the mesh is isotropic, i.e. has the same size in all directions, with a characteristic size $h_{\max }=0.2 \mathrm{~m}$. The half-thickness of the mixture zone is taken as $\varepsilon=0.06 \mathrm{~m}$.

It is important to note here that the choice of the value of the half-thickness $\varepsilon$ is the result of two conditions. The first condition is related to the fact that the artificial mixing zone must be relatively small compared to the characteristic length of the physical problem. The second condition arises from the need for a sufficient number of mesh elements within the mixing zone to ensure a smooth numerical transition of the properties and a good spatial discretisation of the Dirac function. For the latter condition, the thickness is set to approximately three times $h_{\perp}$. The first condition is ensured by mesh refinement in the vicinity of the interface.

Different computations are performed for different values of the resistance $R_{t h}$ ranging from $10^{-5}$ (nearly perfect interface) to $10^{5} \mathrm{~K} \cdot \mathrm{W}^{-1} \cdot \mathrm{m}^{2}$ (nearly insulating interface). Thermal conductivities of materials are $k_{1}=0.1 \mathrm{~W} \cdot \mathrm{m}^{-1} \cdot \mathrm{K}^{-1}$ and $k_{2}=10 \times k_{1}$. The mixed conductivity profile along the $\mathrm{x}$-axis using equation (18) is given in figure 3 for different thermal contact resistance values.

In figure 4 , the computed and the exact temperature fields are plotted versus the $\mathrm{X}$ coordinate. We can observe that the strong temperature jump occurring at high thermal contact resistance is well captured by the proposed numerical model. 


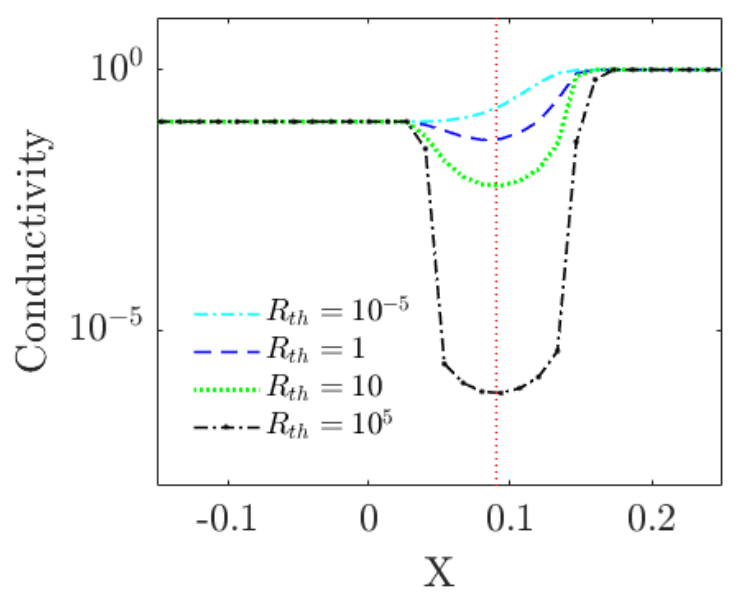

Figure 3: Conductivity profile for different thermal contact resistance values.

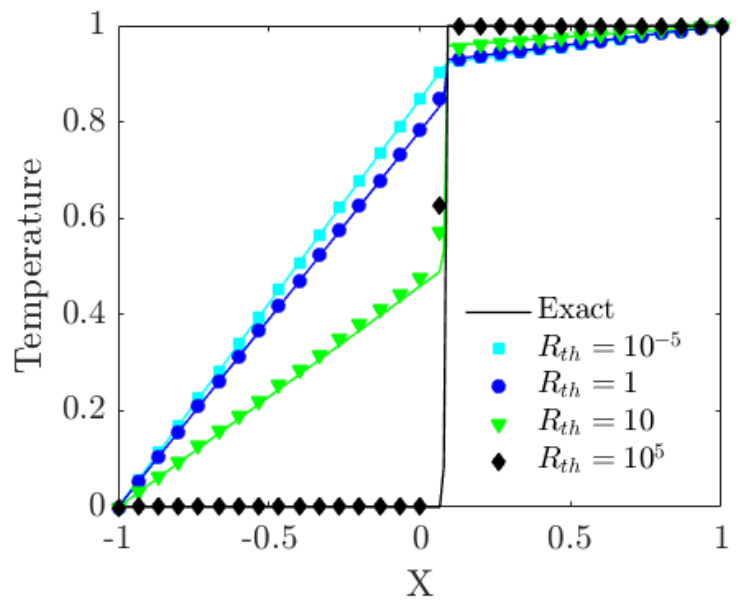

Figure 4: Steady temperature showing the jump at the interface for different thermal contact resistance values

\subsubsection{Spherical interface benchmark}

In the present example schematised in figure 5 , we consider a composite sphere composed of a coating of outer radius $r_{2}$ and a core of radius $r_{1}$ separated by an interface with thermal resistance. The surface of the coating is submitted to a fixed temperature field given by a linear evolution along the z-axis, with temperature gradient $\boldsymbol{G}=\left(0,0, G_{z}\right)$ :

$$
T=\boldsymbol{G} \cdot \boldsymbol{x}=G_{z} \times z
$$

The steady state solution of this configuration is written in the spherical coordinates system $(r, \theta, \phi)$ as:

$$
T(r, \theta)=\left\{\begin{array}{l}
\left(a_{1} r+\frac{b_{1}}{r^{2}}\right) G_{z} \cos (\theta) r<r_{1} \\
\left(a_{2} r+\frac{b_{2}}{r^{2}}\right) G_{z} \cos (\theta) r>r_{1}
\end{array}\right.
$$




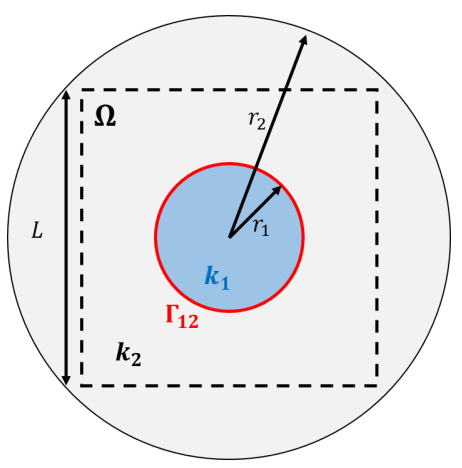

(a) Geometry

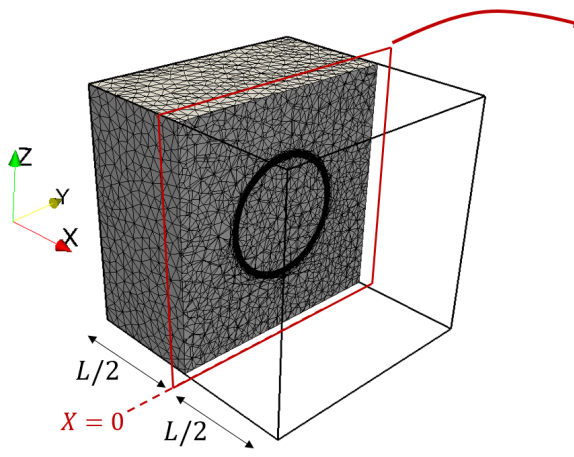

(b) Mesh

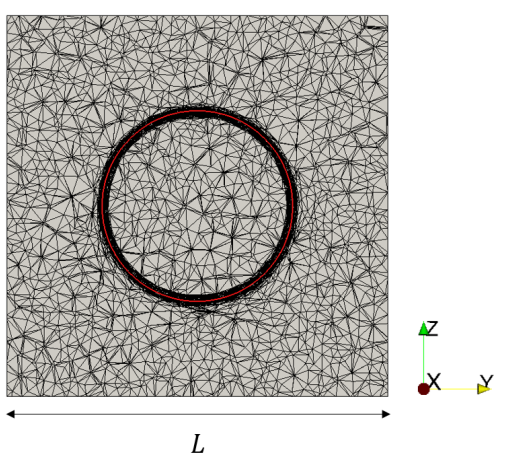

(b) Mesh

Figure 5: Setup of the numerical simulation for a 3D heat flow configuration.

According to the fact that the temperature at the centre must be finite, we necessarily have:

$$
b_{1}=0
$$

Similarly as in section 3.1.1, the boundary condition (28), the continuity of the heat flux across the interface at $r=r_{1}$ and the temperature jump condition are used to finally find:

$$
\begin{gathered}
a_{1}=\frac{3 k_{2} r_{1} r_{2}^{3}}{\beta+\gamma} \\
a_{2}=\frac{\beta}{\beta+\gamma} \\
b_{2}=\frac{r_{2}^{3} \gamma}{\beta+\gamma}
\end{gathered}
$$

where

$$
\begin{gathered}
\beta=\left[k_{1} r_{1}+2 k_{2}\left(r_{1}+k_{1} R_{t h}\right)\right] r_{2}^{3} \\
\gamma=\left[k_{1} k_{2} R_{t h}+\left(k_{2}-k_{1}\right) r_{1}\right] r_{1}^{3}
\end{gathered}
$$

Equation (29) together with (31)-(33) is the analytical solution which will be compared to the numerical solution given by the present model. For that, we consider a cubic computational domain $\Omega$ of a side length size $L=4 r_{1}$ centred with the spheres as shown in figure 5(a). As the outer surface of the coating is not explicitly included in the computational domain $\Omega$, we cannot impose the boundary condition (28) directly. One way to fulfil this condition indirectly is to prescribe the stationary analytical solution as a Dirichlet boundary condition at the borders of the cube, $\partial \Omega$. Simulations are performed with a mesh of approximately $2 \times 10^{5}$ elements. The mesh is refined in a $0.1 \mathrm{~m}$ wide band around the interface as shown in figure $5(\mathrm{~b})$. The size of the elements within the band is $0.008 \mathrm{~m}$ in the direction normal to the interface and $0.1 \mathrm{~m}$ in the tangential direction. Outside this band the elements have a characteristic size of $0.2 m$ in all directions. The half-thickness of the interface is $\varepsilon=0.025 \mathrm{~m}$. Calculations are performed with the set of parameters given in table 1.

The simulated temperature field in the YZ-plane centred with the inner spherical domain is given in figure 6. Values of the thermal contact resistance are varied from 0 to $10^{5} \mathrm{~K} \cdot W^{-1} \cdot \mathrm{m}^{2}$. Figure 7 displays the comparison with the analytical solution of temperature profiles along the z-axis, with $z \geq 0$. Note that 
the solution for $R_{t h}=0 \mathrm{~K} . W^{-1} \cdot \mathrm{m}^{2}$ is not shown in figure 7 as it superimposes with $R_{t h}=1 \mathrm{~K} . W^{-1} \cdot \mathrm{m}^{2}$. For such low values of the heat resistance, the temperature jump at the interface vanishes. This is also shown when comparing figure 6(a) with figure 6(b). Another observation is that for large values of the heat resistance, the temperature in the spherical core is almost uniform. When increasing $R_{t h}$ from $10^{3}$ to $10^{5} \mathrm{~K} . W^{-1} \cdot \mathrm{m}^{2}$, the temperature variation at the interface does no vary much. Finally, one can observe that the temperature fields only differ in the inner spherical core due to the high ratio $k_{2} / k_{1}$, low ratio $r_{2} / r_{1}$ and the Dirichlet boundary condition applied at the boundaries of the coating domain. The temperature profile in the core remains linear, which is also expected under a steady diffusion regime. Once again, the thermal contact resistance model presented in this work shows good comparison with the analytical solution, demonstrating its use in a 3D heat flow configuration with an immersed boundary between domains with different thermal conductivity.
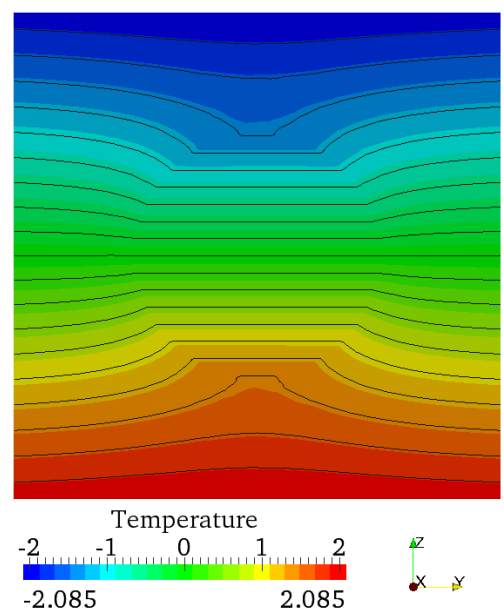

(a) $R_{t h}=0 K . W^{-1} \cdot m^{2}$
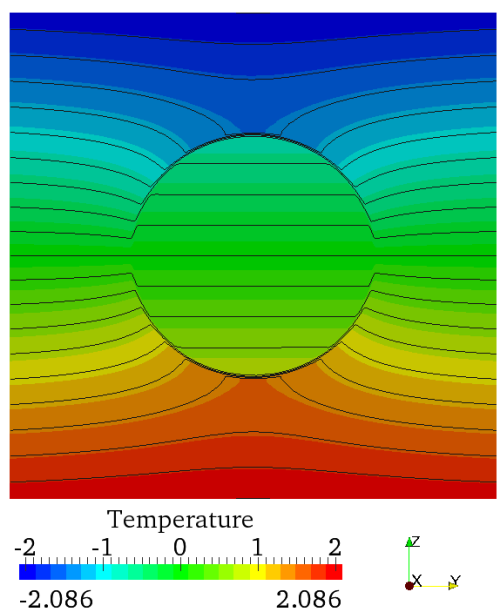

(d) $R_{t h}=10^{2} K \cdot W^{-1} \cdot m^{2}$
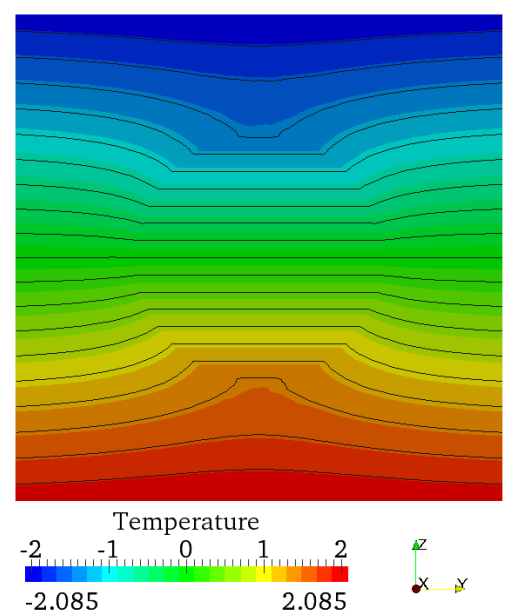

(b) $R_{t h}=1 K \cdot W^{-1} \cdot m^{2}$
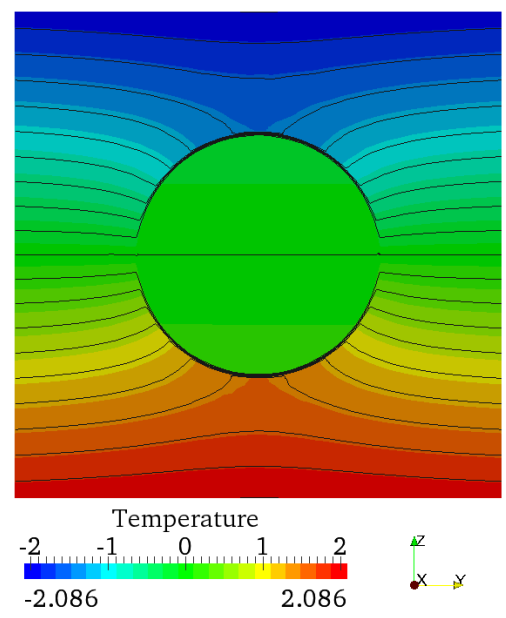

(e) $R_{t h}=10^{3} K \cdot W^{-1} \cdot m^{2}$
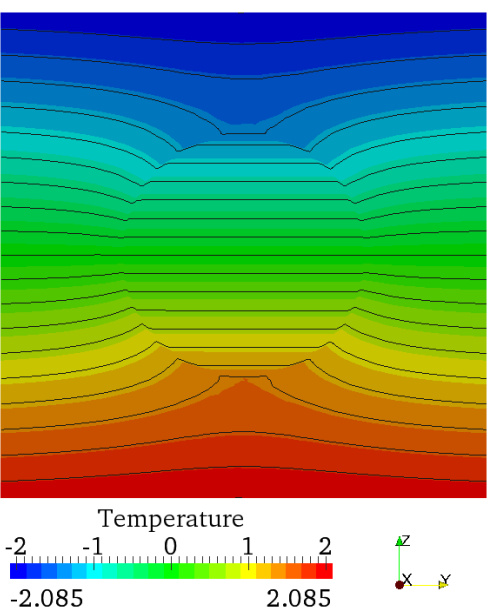

(c) $R_{t h}=10 \mathrm{~K} \cdot W^{-1} \cdot \mathrm{m}^{2}$
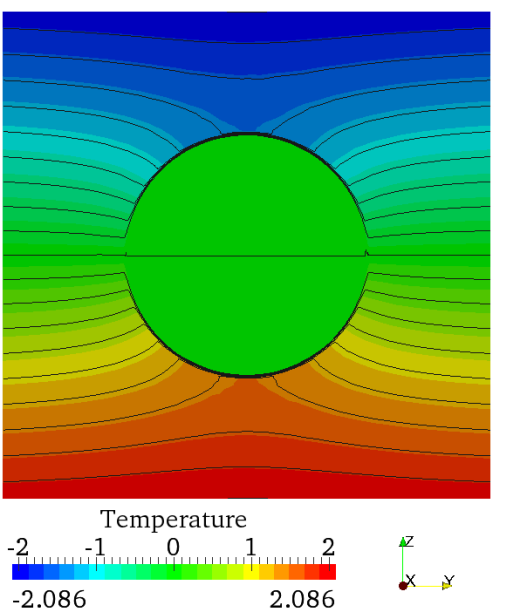

(f) $R_{t h}=10^{5} \mathrm{~K} \cdot W^{-1} \cdot \mathrm{m}^{2}$

Figure 6: Temperature field in YZ-plane through the centre of the simulation domain. 


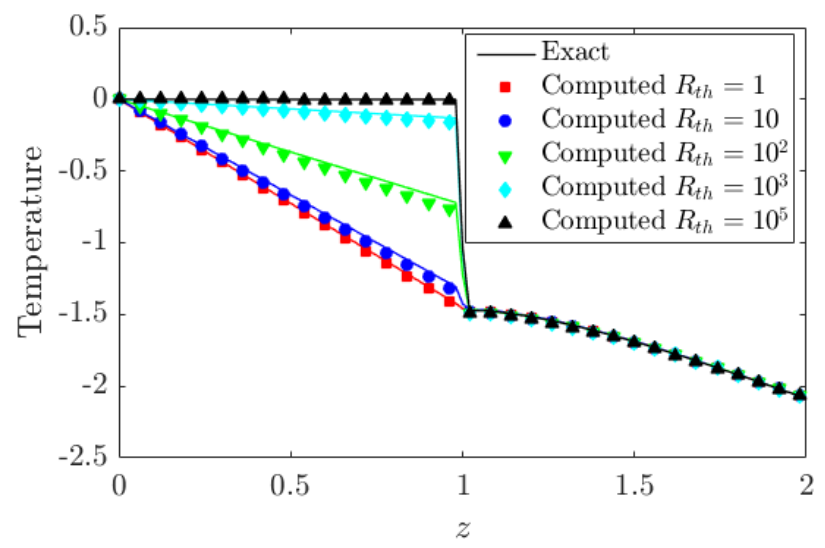

Figure 7: Steady temperature profiles showing the jump at the interface for different thermal contact resistance values.

\begin{tabular}{|l|cccccc|}
\hline Parameter & $G_{z}$ & $r_{1}$ & $r_{2}$ & $k_{1}$ & $k_{2}$ & $R_{t h}$ \\
\hline Value & -1 & 1 & 3 & 0.01 & 1 & {$\left[0,10^{5}\right]$} \\
\hline Unit & $K . m^{-1}$ & $m$ & $m$ & $W . m^{-1} \cdot K^{-1}$ & $W . m^{-1} \cdot K^{-1}$ & $K . W^{-1} \cdot m^{2}$ \\
\hline
\end{tabular}

Table 1: Values of the simulation parameters for results presented in figures 6 and 7 .

\subsection{Unsteady conduction}

In order to evaluate the ability of the new model to handle unsteady heat transfer between two materials including a thermal contact resistance at the interface, we compare the results of the new model (referred to as implicit gap) to the results of a direct numerical simulation of an explicit gap between the two materials (referred to as explicit gap). The geometry of these two cases are shown in figure 8.

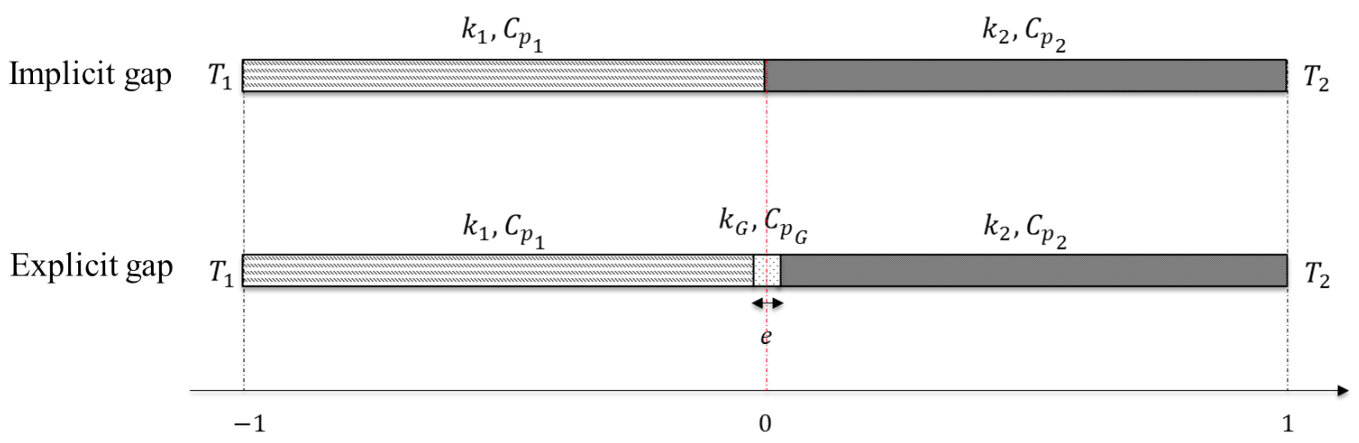

Figure 8: Setup for the unsteady conduction validation test. The reference case is referred to as "Explicit" and the evaluated case is referred to as "Implicit".

The temperature at $x=-1$ and $x=1$ are fixed to $T_{1}=100 K$ and $T_{2}=0 K$ respectively. The top and the bottom boundaries are adiabatic $\left(q_{B}=0\right)$ so heat flux is only one-dimensional. The initial temperature is $T_{0}=0 K$ in the whole domain. The thermal conductivities of each material are $k_{1}=200 \mathrm{~W} \cdot \mathrm{m}^{-1} \cdot \mathrm{K}^{-1}$, $k_{2}=10 \mathrm{~W} \cdot \mathrm{m}^{-1} \cdot K^{-1}$ and $k_{G}=0.1 \mathrm{~W} \cdot \mathrm{m}^{-1} \cdot K^{-1}$. The thickness of the gap is $e=0.002 \mathrm{~m}$. So the thermal contact resistance value is $R_{t h}=e / k_{G}=0.02 \mathrm{~K} . W^{-1} . \mathrm{m}^{2}$.

The meshes in the vicinity of the contacting surfaces are shown for each case in figures $9(\mathrm{a})$ and $9(\mathrm{~b})$. Since the gap width is very small, the mesh used for simulation of the explicit gap is much finer around the contact than that used for the implicit gap. Inside the region of fine mesh, which covers the gap layer, 
the mesh size is $10^{-4} \mathrm{~m}$ in the direction perpendicular to the interface. This is required to ensure enough mesh elements within the gap domain. On the other hand, the thickness of the mixing zone for the implicit case is $2 \varepsilon=0.03 \mathrm{~m}$ so that ratio $2 \varepsilon / e \gg 1$. The mesh size inside the mixing zone is $0.005 \mathrm{~m}$ in the direction perpendicular to the interface. The volumetric specific heat of each material are $C_{p_{1}}=1.5 \times$ $10^{5} \mathrm{~J} . K^{-1} . \mathrm{m}^{-3}$ and $C_{p_{2}}=10^{5} \mathrm{~J} . K^{-1} \cdot \mathrm{m}^{-3}$. Three values of $C_{p_{G}}$ are considered: $C_{p_{G}}=10^{3} \mathrm{~J} \cdot \mathrm{m}^{-3} \cdot K^{-1}$, $C_{p_{G}}=1.3 \times 10^{5} \mathrm{~J} . \mathrm{m}^{-3} \cdot K^{-1}$ and $C_{p_{G}}=10^{7} \mathrm{~J} \cdot \mathrm{m}^{-3} \cdot K^{-1}$, corresponding to $C_{p_{G}} \ll \bar{C}_{p}, C_{p_{G}}=\mathcal{O}\left(\bar{C}_{p}\right)$ and $C_{p_{G}} \gg \bar{C}_{p}$, respectively. For each value of $C_{p_{G}}$ two formulae of the volumetric specific heat mixing law are tested, referred to as Formula 1 given by equation (22) and Formula 2 given by equation (11). Results are shown in figures 10, 11 and 12 as temperature profiles along the x-axis for both the (a) explicit and (b) implicit gap.

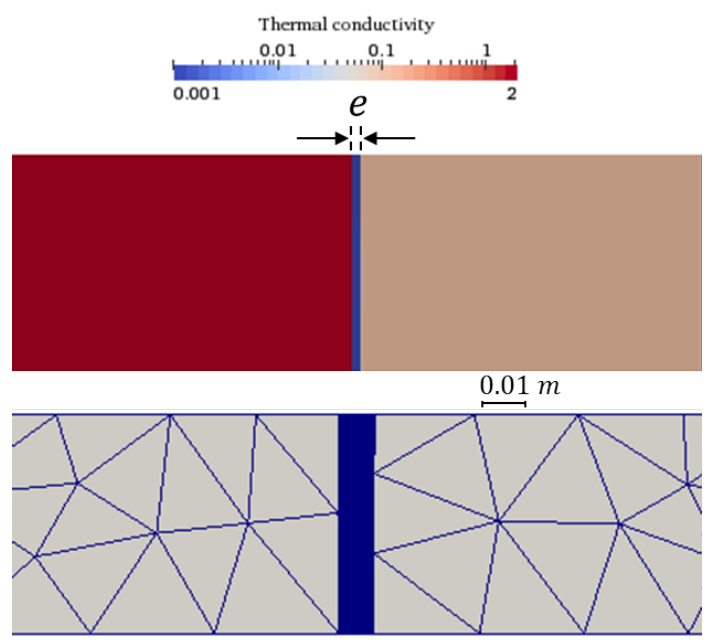

(a) Explicit gap

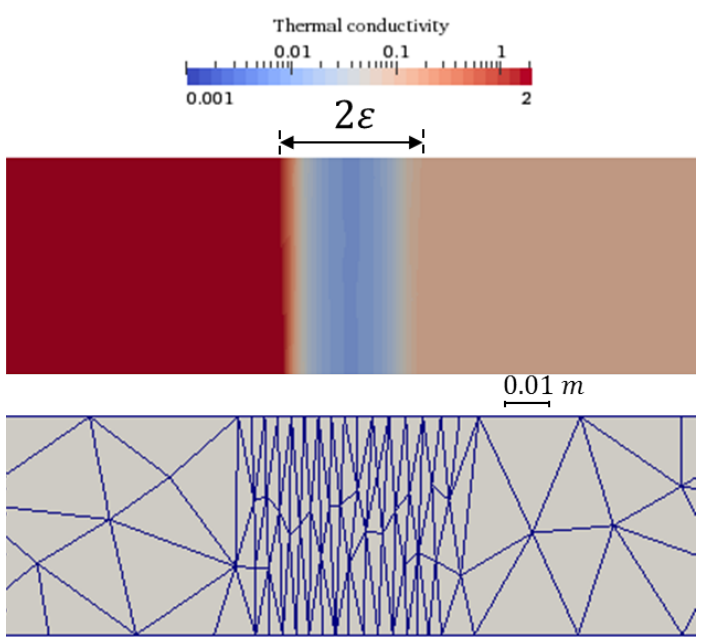

(b) Implicit gap

Figure 9: Mesh size and distribution of the thermal conductivity for the (a) explicit and (b) implicit gap configurations.

In figure 10, when $C_{p_{G}} \ll \bar{C}_{p}$, the volumetric specific heat mixing law for a perfect contact, i.e. using Formula 1 with equation (11), is sufficient to describe the transient heat conduction, i.e. to retrieve the more exact numerical solution given by (plain curves) the explicit gap formulation. The same can be observed in figure 11 when $C_{p_{G}}=\mathcal{O}\left(\bar{C}_{p}\right)$. No or very little difference is revealed between symbols and curves. However, when $C_{p_{G}} \gg \bar{C}_{p}$, figure 12 reveals differences between Formula 1 and the more precise Formula 2. This does not concern the steady behaviours when the time is large enough and two linear temperature evolution have settled in the domains (time $t=8000 \mathrm{~s}$ ), but intermediate times for unsteady heat flows. For instance, at time $t=600 \mathrm{~s}$, a clear difference appears in figure 12(a) between the explicit gap configuration, that corresponds to the reference situation, and the implicit configuration using Formula 1 with equation (11). A gap with a high volumetric specific heat compared to the surrounding materials is thus better described with the Formula 2 using the mixing law given by equation (22) as shown in figure12(b). Despite this observation that reveals the consequences of using the volumetric specific heat mixing law for a perfect contact, it is clear that using equation (22) does not require big additional efforts while reaching excellent agreement when dealing with the prediction of non-stationary heat conduction. 


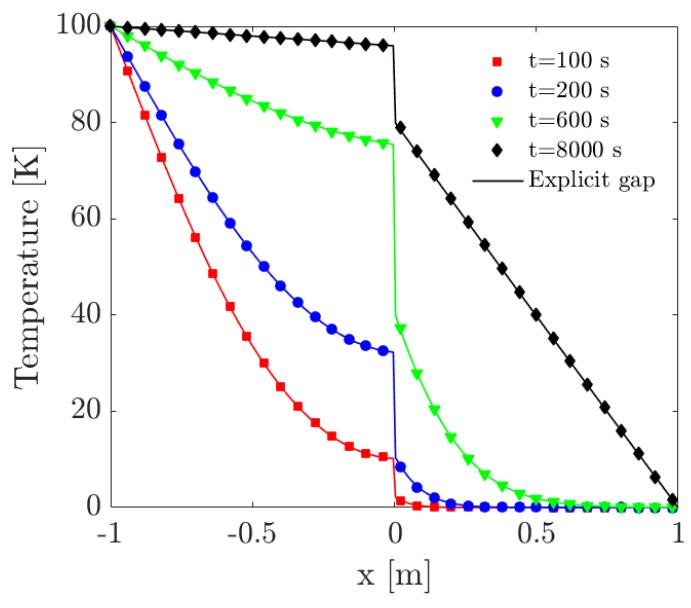

(a) Formula 1: equation (11)

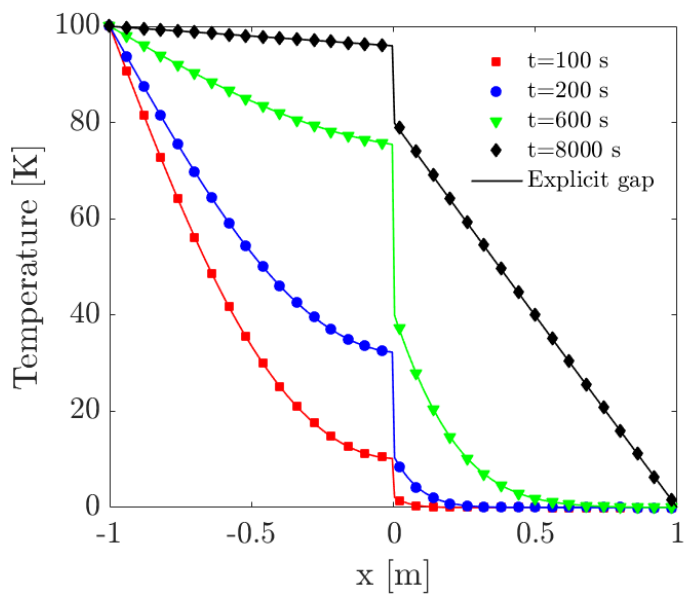

(b) Formula 2: equation (22)

Figure 10: Time-evolution of the temperature jump across the interface for $C_{p_{G}} \ll \bar{C}_{p}$

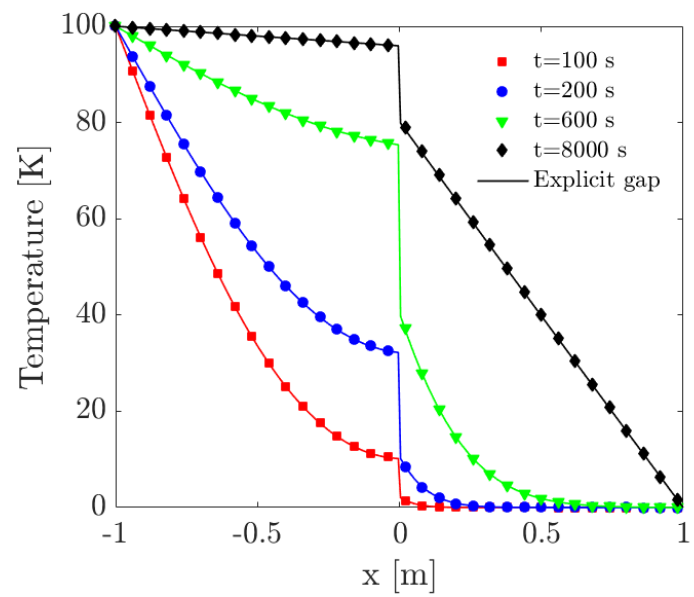

(a) Formula 1: equation (11)

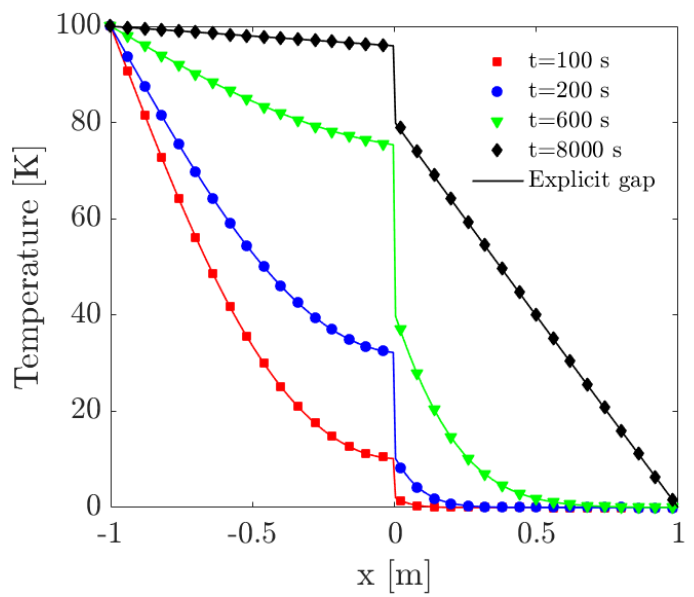

(b) Formula 2: equation (22)

Figure 11: Time-evolution of the temperature jump across the interface for $C_{p_{G}}=\mathcal{O}\left(\bar{C}_{p}\right)$ 


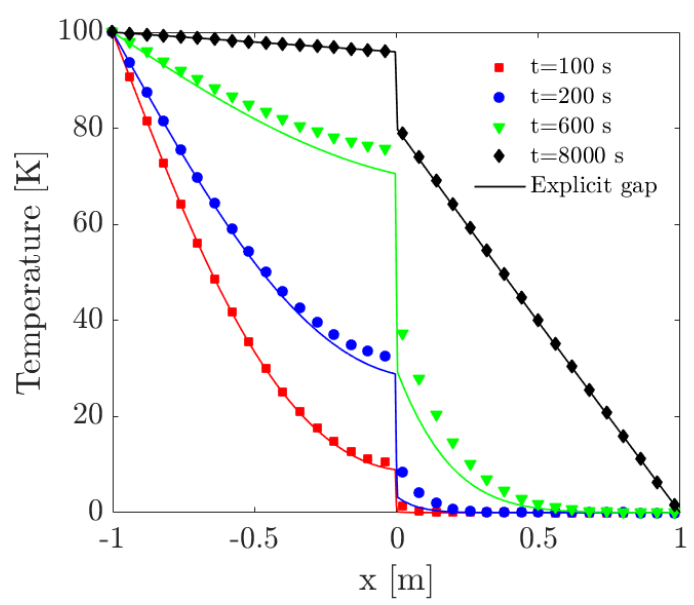

(a) Formula 1: equation (11)

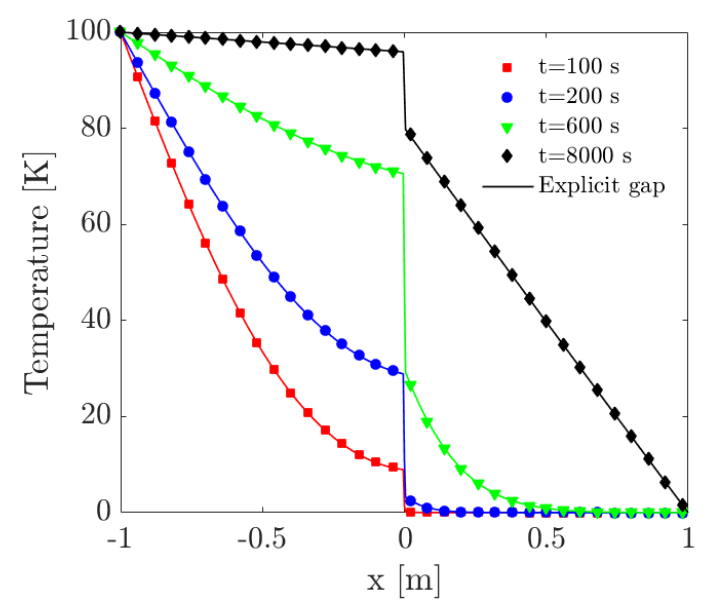

(b) Formula 2: equation (22)

Figure 12: Time-evolution of the temperature jump across the interface for $C_{p_{G}} \gg \bar{C}_{p}$

\subsection{Application to chill cooling experiment of a steel sample on board the International Space Station}

One of the many industrial processes where thermal contact resistance plays an important role is the continuous casting of steels. Indeed, due to the roughness of the mould surface, tiny pockets of gas trapped at the boundary between the mould and the metal can form a resistance to heat transfer. Hence, the thermal contact resistance may compromise the performance of the process.

The ESA-MAP project entitled CCEMLCC (for Chill Cooling for the ElectroMagnetic Levitator in relation with $\underline{\text { Continuous }}$ Casting of steel ) aims at enhancing the understanding of solidification of steel products, more particularly, the defects formed during casting process [24]. For this purpose, the solidification of an initially freely suspended molten steel droplet in microgravity condition is investigated. The mould of the continuous casting machine is modelled by the use of a ceramic chill plate which extracts the heat from the sample and thus triggers nucleation of the solid phase. The motivation for conducting experiments in microgravity is to reduce the effects of gravity-related sources of mass and heat transfers and to determine how gravity-independent phenomena can influence the solidification process.

Several steel grades have been tested in this project. We focus in this paper on the sample Fe-0.9C-0.26Si (composition in $w t . \%$ ) because this steel sample has been processed both during parabolic flights and on board the International Space Station (ISS). The main finding of the chill cooling tests is the elongation of the solidifying droplet in the direction of the chill plate axis. The solidified droplet in the ISS is not brought back to Earth at the time of writing this paper for analysis, but the same sample processed during the parabolic flights showed that the contact surface of the sample with the chill plate is flat but not perfectly smooth as shown in Figure 13(b). The latter observation raises the question of the influence of the thermal contact resistance on the cooling rate. To answer this question, we apply the present model to simulate the chill cooling and solidification of the steel sample.

We only focus hereafter on solidification governed by heat conduction from the droplet to the chill plate without convective heat transport in the bulk liquid metal (no flow is considered) and without radiative heat transfer between the droplet and the chill. The modelling of heat transfer with solidification relies on a temperature-based energy solver able to handle tabulated properties for all phase $\varphi$ (the liquid phase $(l)$ and all possible individual solid phases $(s)$ ) involved during solidification and solid-state transformations as a function of temperature $T$. For more details about this non-linear energy solver coupled with tabulated thermodynamic properties, we refer the reader to the following paper [26]. The monolithic formulation of 


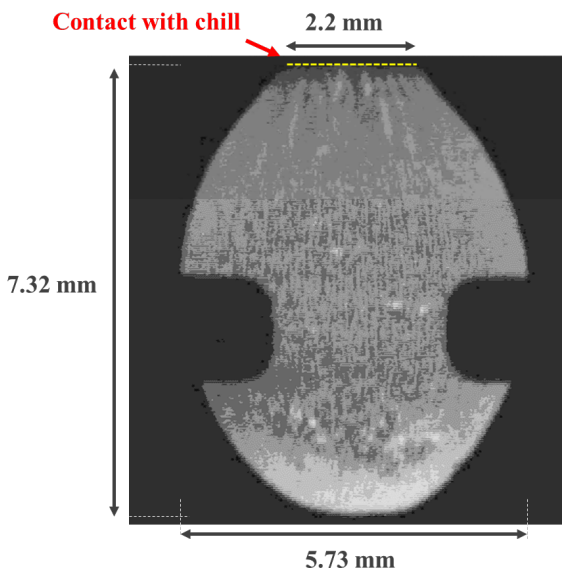

(a)

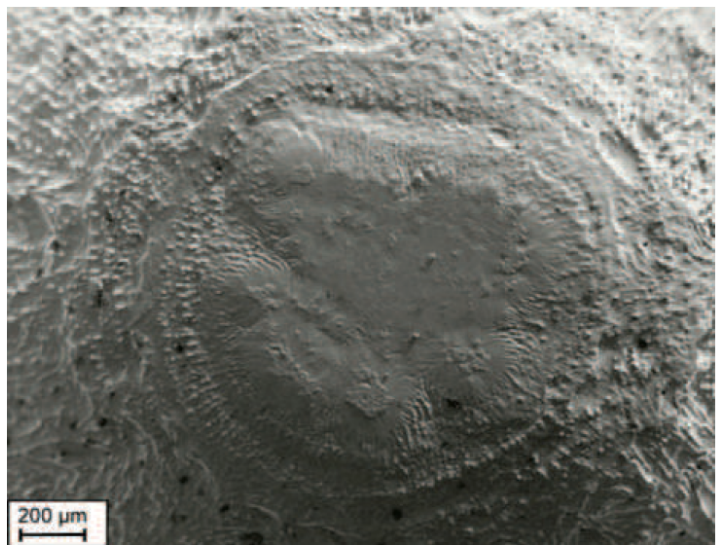

(b)

Figure 13: (a) The final shape of the solidified sample after processing on board the ISS [25]. (b) Scanning Electron Microscopy imaging of the contact area of the solidified sample processed during a parabolic flight on board a zero-g airplane [24]

the energy conservation equation in a computational domain containing the metallic sample $(\mathrm{M})$ in contact with the chill plate $(\mathrm{C})$ together surrounded by Argon gas (A) writes:

$$
\int_{\Omega} \frac{\partial\langle\rho h\rangle}{\partial t} w d V+\int_{\Omega} k \nabla T \cdot \nabla w d V=0
$$

where $\langle\rho h\rangle$ is the mixed field of the averaged volumetric enthalpies $\langle\rho h\rangle_{\Omega_{i}}, \Omega_{i} \in\{M, A, C\}$, of all subdomains using the smooth Heaviside functions $H_{\Omega_{i} \in\{M, A, C\}}$ such as:

$$
\langle\rho h\rangle=\langle\rho h\rangle_{M} H_{M}+\langle\rho h\rangle_{C} H_{C}+\langle\rho h\rangle_{A} H_{A}
$$

Considering a representative volume element, the average volumetric enthalpy of the metal writes:

$$
\langle\rho h\rangle_{M}=\sum_{\varphi \in M} g^{\varphi}\langle\rho\rangle^{\varphi}\langle h\rangle^{\varphi}
$$

The volume fractions $g^{\varphi}$, the densities $\langle\rho\rangle^{\varphi}$ and the enthalpies $\langle h\rangle^{\varphi}$ are tabulated with respect to temperature $T$. As for the chill plate, made only of a solid ceramic phase $S i_{3} N_{4}$, the average volumetric enthalpy is a linear function of the temperature, $\langle\rho h\rangle_{C}=C_{p_{C}} . T$ where $C_{p_{C}}=2.303 \times 10^{6} J \cdot K^{-1} \cdot m^{-3}$. The same principle applies to the argon gas enthalpy $\langle\rho h\rangle_{A}=C_{p_{A}} \cdot T$ with $C_{p_{A}}=1300 \mathrm{~J} \cdot \mathrm{K}^{-1} \cdot \mathrm{m}^{-3}$.

Following the equation (25), the mixed thermal conductivity in this multi-material domain writes:

$$
k=\left(\frac{H_{M}}{k_{M}}+\frac{H_{A}}{k_{A}}+\frac{H_{C}}{k_{C}}+R_{t h M C} \delta_{M C}+R_{t h M A} \delta_{M A}+R_{t h A C} \delta_{A C}\right)^{-1}
$$

where $k_{M}=42 \mathrm{~W} \cdot \mathrm{m}^{-1} \cdot \mathrm{K}^{-1}, k_{C}=50 \mathrm{~W} \cdot \mathrm{m}^{-1} \cdot \mathrm{K}^{-1}$ and $k_{A}=0.01 \mathrm{~W} \cdot \mathrm{m}^{-1} \cdot \mathrm{K}^{-1}$ are the thermal conductivities of the metal, chill and gas respectively, taken at room temperature and considered constant. $\delta_{M C}$, $\delta_{M A}$ and $\delta_{A C}$ are the smooth Dirac functions associated to the metal-chill interface $\Gamma_{M C}$, the metal-gas interface $\Gamma_{M A}$ and the chill-gas interface $\Gamma_{A C}$ respectively. The interfaces $\Gamma_{M A}$ and $\Gamma_{A C}$ involve a full contact with the gas. Therefore, the thermal contact is assumed to be perfect at these latter boundaries, i.e. $R_{t h M A}=R_{t h A C}=0$. A thermal contact resistance is only applied at the metal-chill interface $\Gamma_{M C}$. 
For the sake of simplicity, $R_{t h M C}$ is simply denoted by $R_{t h}$. Consequently, the mixed thermal conductivity becomes:

$$
k=\left(\frac{H_{M}}{k_{M}}+\frac{H_{A}}{k_{A}}+\frac{H_{C}}{k_{C}}+R_{t h} \delta_{M C}\right)^{-1}
$$

It is worth noting that in this experiment any micro-cavities (gap) that may exist between the metal and the chill are probably filled with gas (or vacuum). Therefore, the volumetric specific heat of the gap $C_{p_{G_{M C}}} \in\left[0, \mathcal{O}\left(10^{3}\right)\right]$ at the interface $\Gamma_{M C}$ is negligible compared to the mean volumetric specific heat of the metal and the ceramic $\frac{C_{p_{M}}+C_{p_{C}}}{2}=\mathcal{O}\left(10^{6}\right)$. According to the equation (26) and the discussion in section 3.2 , as $C_{p_{G_{M C}}} \ll \frac{C_{p_{M}}+C_{p_{C}}}{2}$ and $C_{p_{G_{M A}}}=C_{p_{G_{A C}}}=0$, the mixed volumetric specific heat is:

$$
C_{p}=C_{p_{M}} H_{M}+C_{p_{C}} H_{C}+C_{p_{A}} H_{A}
$$

In terms of volumetric enthalpy, this is equivalent to writing the equation (37).

In this problem, we need two level-set functions to compute the Heaviside and Dirac functions: one level set $\phi_{M}$ to describe the boundary of the droplet and the second $\phi_{C}$ for the chill plate boundary. The droplet shape is based on the final shape of the solidified sample on board the ISS shown in Figure 13(a). It is approximated to an ellipsoid of minor horizontal axis $5.73 \mathrm{~mm}$ and major vertical axis $7.65 \mathrm{~mm}$. This ellipsoid is truncated from the top so that the droplet is $7.31 \mathrm{~mm}$ high and the plane surface in contact with the chill is a disc $2.2 \mathrm{~mm}$ diameter. Note that the final shape of the solidified sample processed on board a zero-g airplane has slightly different dimensions, particularly smaller contact area with the chill as observed in Figure 13(b). The chill plate is a cylinder of $16 \mathrm{~mm}$ diameter and $3 \mathrm{~mm}$ high as in the ISS experiment. The Heaviside functions $H_{M}$ and $H_{C}$ are determined by formula (10) using $\phi_{M}$ and $\phi_{C}$ respectively. The gas Heaviside is then $H_{A}=1-H_{M}-H_{C}$. The mixing half-thickness $\epsilon$ is fixed to $0.18 \mathrm{~mm}$. The Dirac function $\delta_{M C}$ is calculated as:

$$
\delta_{M C}=\left\{\begin{array}{lll}
\delta_{M} & \text { if } & H_{C}>0 \\
0 & \text { if } & H_{C}=0
\end{array}\right.
$$

where $\delta_{M}$ is the metal Dirac function defined by equation (19) using $\phi_{M}$.

The computational domain is a box of a side length of $20 \mathrm{~mm}$ in the vertical Z-direction and $28 \mathrm{~mm}$ in the $\mathrm{X}$ - and Y-directions (Figure 14(a)). The mesh size around the interfaces is $0.06 \mathrm{~mm}$ in the direction of the normal to the interface. Inside each domain, the mesh size is $0.2 \mathrm{~mm}$ for the metal, $0.3 \mathrm{~mm}$ for the chill and $0.6 \mathrm{~mm}$ for the gas (Figure 14(b)).

The initial temperature is $1921 \mathrm{~K}$ for the metal and the gas and $300 \mathrm{~K}$ for the chill plate. The adiabatic condition is considered at the boundaries of the computational domain. Several simulations were performed for different values of thermal contact resistance $R_{t h} \in\left\{0,10^{-5}, 2 \times 10^{-5}, 5 \times 10^{-5}, 10^{-4}\right\} K . W^{-1} . m^{2}$. The comparison with the experiment is based on the time-evolution of the solidification front position from the chill plate. The experimental curve was obtained from image processing of the video recorded with an embedded high-speed camera in the experimental device on board the ISS. The solidification front was extracted from the recorded images using the colour contrast between the solid and liquid phases visible on the free surface of the sample. Theoretically, the solidification front is located at the boundary of the solid dendrites with the liquid phase. This interface forms at the liquidus temperature $T_{L}=1739 \mathrm{~K}$. The numerical results with different $R_{t h}$ values for $T=T_{L}$ are shown in figure 15(a). With a perfect contact ( $R_{t h}=0$, i.e. no model for thermal contact resistance), it takes 4 seconds for the liquidus iso-value to reach the bottom of the sample. The experiment shows that the front reaches the bottom of the droplet after approximately 10 seconds. When the thermal contact resistance is taken into account in the simulations, the solidification front growth slows down. A value of $R_{t h}=10^{-4} K \cdot W^{-1} \cdot m^{2}$ allows getting closer to the experimental solidification duration. However, the experiment shows that the "contrast-based" front is propagating with 


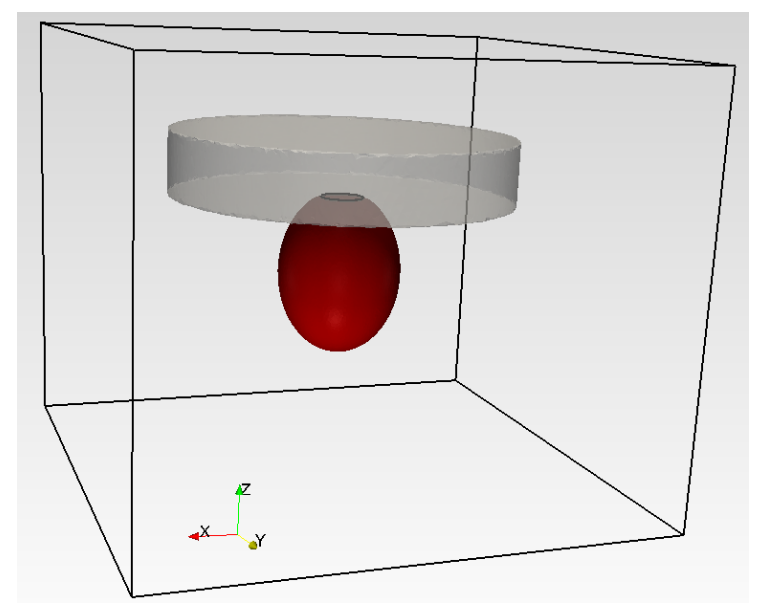

(a) Geometries

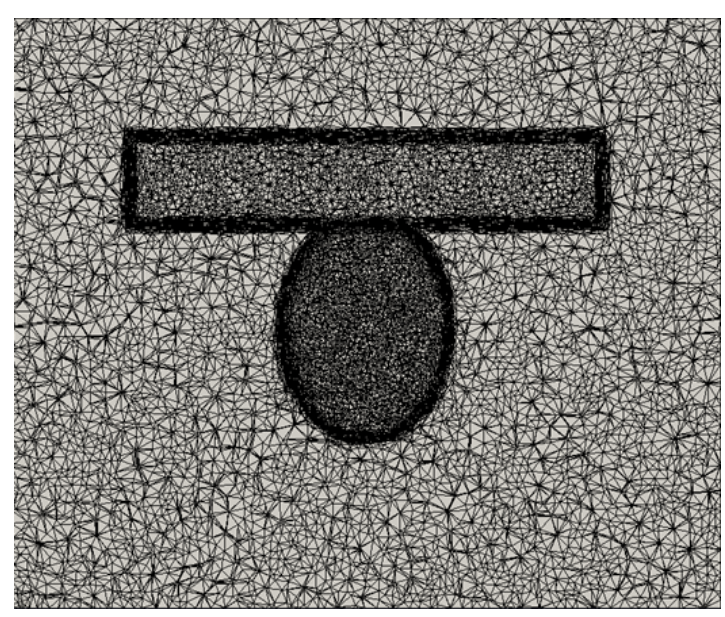

(b) Mesh (cross section)

Figure 14: The setup of the simulation for chill cooling and solidification of a deformed steel droplet showing (a) the geometries of the droplet and chill plate inside the computational domain and (b) a cross section through the 3D finite element mesh

a constant speed. The simulation shows a positive acceleration of the growth of the liquidus-based front. Several parameters could be at the origin of the difference: the fluid flow, the deformation of the droplet but also the difference in the criterion for tracking the front. Indeed, the contrast between the liquid and solid phases on the images is only visible when the solid fraction $g^{s}$ is higher than a certain value. When we choose, as a criterion for front detection, the iso-value $g^{s}=20 \%$ the time-evolution of the front position becomes slower than the liquidus-based front 15(b). In this case, the value of $R_{t h}=2 \cdot 10^{-5} \mathrm{~K} \cdot \mathrm{W}^{-1} \cdot \mathrm{m}^{2}$ gives a good prediction of the experimental behaviour during the first 6 seconds but predicts a shorter solidification duration than observed experimentally.

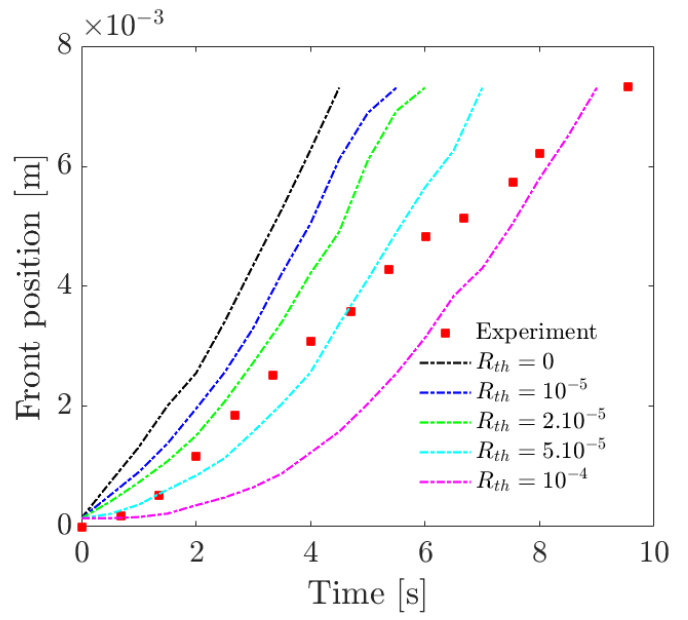

(a) $T=T_{L}$

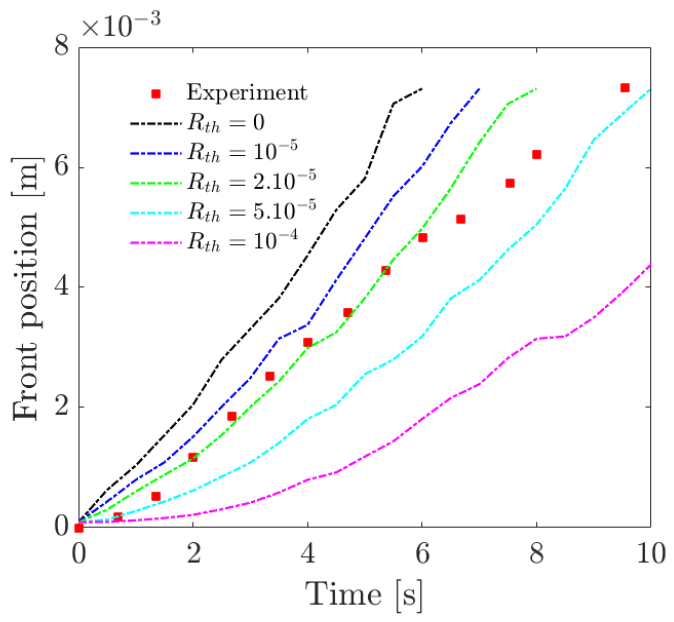

(b) $g^{s}=20 \%$

Figure 15: The time-evolutions of the front position for different thermal contact resistance compared to the experiment

We are aware that the simulation of solidification by pure conduction in a static drop is far from being representative of what really occurs in the experiment. Indeed, fluid flow can play an important role in 
the distribution of heat and chemical species in the liquid metal. In addition, since the chill is exposed to radiative emissions from the sample, its temperature may increase further. This could influence the growth kinetics of the solidification front. Simulations with comprehensive modelling of the physics governing this experiment would give a more accurate thermal resistance value. Nevertheless, we believe that the simulations presented in this work give a first estimation of the order of magnitude of the contact thermal resistance value. Simulation of chill cooling and solidification including convective heat transfer induced by solidification shrinkage and capillary forces present at the metal-gas interface is foreseen and will be the topic of a forthcoming publication.

\section{Conclusion}

A simple and efficient numerical model is proposed to account for thermal contact resistance at an arbitrarily-shaped interface while using a monolithic solution with a diffuse interface. The present model describes the interface by starting from the level-set method and smoothing the transition from one material to the other by a smoothed Heaviside function, hence defining a diffuse interface. The accuracy and robustness of the model are assessed through benchmarks for both steady and transient diffusion regimes. The results and comparisons with the references show that the model can produce a good prediction of the temperature jump across the interface. An application of the present model is shown through simulation of chill cooling experiments of steel samples conducted with the Electromagnetic Levitator facility onboard the International Space Station.

\section{Acknowledgements}

This work was supported by the European Space Agency (Noordwijk, NL) under the projects NEQUISOL (contract number 15236/02/NL/SH) and CCEMLCC (contract number 20277/06/NL/VJ).

\section{References}

[1] E. Swartz, R. O. Pohl, Thermal boundary resistance, Reviews of Modern Physics 61 (1989) 605-668.

[2] A. Hamasaiid, M. Dargusch, T. Loulou, G. Dour, A predictive model for the thermal contact resistance at liquid-solid interfaces: Analytical developments and validation, International Journal of Thermal Sciences 50 (2011) 1445-1459.

[3] H. Prajapati, D. Ravoori, R. Woods, A. Jain, Measurement of anisotropic thermal conductivity and inter-layer thermal contact resistance in polymer fused deposition modeling (FDM), Additive Manufacturing 21 (2018) 84 - 90.

[4] M. Grujicic, C. Zhao, E. Dusel, The effect of thermal contact resistance on heat management in the electronic packaging, Applied Surface Science 246 (2005) 290 - 302.

[5] T. Chen, D. Chen, B. Sencer, L. Shao, Molecular dynamics simulations of grain boundary thermal resistance in UO2, Journal of Nuclear Materials 452 (2014) $364-369$.

[6] L. Steg, H. Lew, Chapter 32 - Hypersonic Ablation, in: W. Nelson (Ed.), The High Temperature Aspects of Hypersonic Flow, vol. 68, Elsevier, $629-680,1964$.

[7] D. Baker, D. Assanis, A methodology for coupled thermodynamic and heat transfer analysis of a diesel engine, Applied Mathematical Modelling 18 (1994) 590 - 601.

[8] C. P. Hallam, W. D. Griffiths, A model of the interfacial heat-transfer coefficient for the aluminum gravity die-casting process, Metallurgical and Materials Transactions B 35 (2004) 721-733.

[9] A. Bendada, A. Derdouri, M. Lamontagne, Y. Simard, Analysis of thermal contact resistance between polymer and mold in injection molding, Applied Thermal Engineering 24 (2004) 2029 - 2040.

[10] K. Pericleous, K. Chan, M. Cross, Free Surface flow and heat transfer in cavities: The SEA algorithm, Numerical Heat Transfer, Part B: Fundamentals 27 (1995) 487-507.

[11] G. Storr, M. Behnia, Comparisons between experiment and numerical simulation using a free surface technique of freefalling liquid jets, Experimental Thermal and Fluid Science 22 (2000) $79-91$.

[12] R. Fedkiw, T. Aslam, B. Merriman, S. Osher, A Non-oscillatory Eulerian Approach to Interfaces in Multimaterial Flows (the Ghost Fluid Method), Journal of Computational Physics 152 (1999) 457 - 492.

[13] J. Principe, R. Codina, A numerical approximation of the thermal coupling of fluids and solids, International Journal for Numerical Methods in Fluids 59 (2009) 1181 - 1201 
[14] E. Hachem, T. Kloczko, H. Digonnet, T. Coupez, Stabilized finite element solution to handle complex heat and fluid flows in industrial furnaces using the immersed volume method, International Journal for Numerical Methods in Fluids 68 (2012) 99-121.

[15] D. Hasselman, L. Johnson, Effective thermal conductivity of composites with interfacial thermal barrier resistance, Journal of Composite Materials 21 (1987) 508-515.

[16] A. Jain, K. K. Tamma, Parabolic Heat Conduction Specialized Applications Involving Imperfect Contact Surfaces: Local Discontinuous Galerkin Finite Element Method-Part 2, Journal of Thermal Stresses 33:4 (2010) 344-355.

[17] J. Yvonnet, Q.-C. He, Q.-Z. Zhu, J.-F. Shao, A general and efficient computational procedure for modelling the Kapitza thermal resistance based on XFEM, Computational Materials Science 5 (2011) 1220-1224.

[18] J. Liu, S. Gu, E. Monteiro, Q. He, A versatile interface model for thermal conduction phenomena and its numerical implementation by XFEM, Computational Mechanics 4 (2014) 825-843.

[19] S. Torquato, M. Rintoul, Effect of the Interface on the Properties of Composite Media, Physical Review Letter 75 (1995) 4067-4070.

[20] R. Lipton, B. Vernescu, Composites with imperfect interface, Proceedings of the Royal Society of London A 452.

[21] C.-W. Nan, R. Birringer, Effective thermal conductivity of particulate composites with interfacial thermal resistance, Journal of Applied Physics 81 (1997) 6692-6699.

[22] H. L. Duan, B. Karihaloo, Effective thermal conductivities of heterogeneous media containing multiple imperfectly bonded inclusions, Physical Review B 75 (2007) 064206.

[23] S. Patankar, Numerical heat transfer and fluid flow, Series on Computational Methods in Mechanics and Thermal Science, Hemisphere Publishing Corporation (CRC Press, Taylor \& Francis Group), 1980.

[24] C.-A. Gandin, M. Bellet, A. Saad, D. Herlach, T. Volkmann, C. Karrasch, V. Uhlenwinkel, N. Ellendt, N. Cifici, G. Zepon, H. Henein, P. D. Khatibi, J. Valloton, P. Siitonen, F. Costes, M. Antonicelli, M. Bobadilla, B. Rivaux, Project ESA-MAP CCEMLCC phase \#2 - Final Report, 2014.

[25] C.-A. Gandin, M. Bellet, G. Guillemot, E. Hachem, A. Aalilija, S. Zhang, T. Volkmann, C. Kreischer, H. Henein, J. Valloton, B. Rivaux, F. Costes, E. Perchat, H. Combeau, I. Poirtrault, M. Cherif, L. Carroz, J. Demurger, V. Descotes, L. Collins, Chill Cooling for the ElectroMagnetic Levitator in relation with Continuous Casting of steels (CCEMLCC) Final Report, 2020.

[26] A. Saad, C.-A. Gandin, M. Bellet, Temperature-based energy solver coupled with tabulated thermodynamic properties Application to the prediction of macrosegregation in multicomponent alloys, Computational Materials Science 99 (2015) $221-231$. 